\title{
A Case of Idiopathic Sequential Profound Bilateral Sudden Sensorineural Hearing Loss Occurring 37 Days After Unilateral Presentation
}

\author{
Joo Young Woo, Chang Lok Ji, Geun Hyung Park, and Byungwoo Yoon \\ Department of Otorhinolaryngology, Busan St. Mary's Hospital, Busan, Korea
}

Received April 1,2021

Revised June 5, 2021

Accepted June 12, 2021

\author{
Address for correspondence \\ Byungwoo Yoon, MD, PhD \\ Department of Otorhinolaryngology, \\ Busan St. Mary's Hospital, \\ 25-14 Yongho-ro 232beon-gil, \\ Nam-gu, Busan 48575, Korea \\ Tel $+82-51-933-7222$ \\ Fax +82-51-232-8600 \\ E-mail wooya8207@gmail.com
}

\begin{abstract}
Sudden sensorineural hearing loss (SSNHL) is a common disorder; however, sequential, bilateral presentation of the disease is rarer than unilateral presentation. Clinical otologists usually focus on treating the side with impaired hearing when patients first present with unilateral SSNHL, and therefore, may not warn patients of the possibility of subsequent hearing impairment in the contralateral ear. Furthermore, it is professionally discouraging when a patient presents with profound, sequential SSNHL after initial treatment. This may adversely impact the doctor-patient relationship, even if the patient is offered the best possible care from their first visit. Herein, we report the case of a patient with profound, idiopathic, bilateral SSNHL with a time interval of 37 days between involvement of both ears. Even though high-dose steroids were administered intraorally and intratympanically, the patient's hearing was not restored, and the patient eventually required bilateral cochlear implant surgery. Our report demonstrates that sequential, profound, bilateral SSNHL may manifest without any specific signs.
\end{abstract}

J Audiol Otol 2021;25(4):235-240

Keywords: Hearing loss, sudden; Hearing loss, bilateral.

\section{Introduction}

The incidence of sudden sensorineural hearing loss (SSNHL) is estimated to be between 5 and 30 cases per 100,000 individuals per year [1]. Bilateral SSNHL (BSSNHL) is relatively rare compared to unilateral SSNHL (USSNHL) and accounts for $<5 \%$ of all cases. According to the time interval between the onset of SSNHL in the first affected ear and that in the subsequent ear, BSSNHL is generally categorized into two types: simultaneous ( $<3$ days) and sequential ( $3-30$ days), although some authors have also labeled cases with $>30$ days interval as sequential [2-5].

BSSNHL has been mostly associated with serious systemic pathologies rather than with an idiopathic etiology [6]. Therefore, it is not easy to anticipate the occurrence of sequential BSSNHL when a patient presents at an otology outpatient practice with USSNHL, especially in those who do not have

This is an Open Access article distributed under the terms of the Creative Commons Attribution Non-Commercial License (https://creativecommons.org/licenses/by-nc/4.0/) which permits unrestricted non-commercial use, distribution, and reproduction in any medium, provided the original work is properly cited. a relevant past history. Otologists tend to focus on treating the hearing loss on the impaired side and may forget to caution the affected patient regarding the possibility of development of hearing impairment in the currently uninvolved side. Recurrent presentation of such a patient to the otologist with serial, profound BSSNHL, now also involving the contralateral ear, is professionally discouraging due to the high morbidity associated with discomfort during verbal communication, and it may result in the patient losing confidence in the treating doctor.

Here, we report the case of a patient who experienced profound, idiopathic, bilateral SSNHL with a time interval of 37 days between the involvement of both ears, discuss possible causes of the presenting symptoms, and review associated literature on this topic.

\section{Case Report}

A 75-year-old woman visited our clinic three times for sudden hearing loss associated with tinnitus and vertigo. She had concurrent hypertension and hyperlipidemia but denied a 
history of head injury, autoimmune disease, meningitis, cardiac symptoms, and other relevant illnesses. She had no specific occupational history. The patient was prone to anxiety, took diazepam whenever she felt dizzy, and noticed temporal high-blood pressure readings during self-measurements. She had a habit of daily caffeine intake and of taking vitamin supplements. During her first visit to our center, she reported that she experienced vertigo without hearing loss, which lasted for a few minutes, followed intermittently by a sensation of disequilibrium. She also experienced nausea and denied any other associated symptoms. She did not present with spontaneous nystagmus. However, on video-guided eye movement examination, she showed subjective symptoms and torsional nystagmus at the left Dix-Hallpike position (Fig. 1). Diffusion-weighted magnetic resonance imaging (MRI) showed no acute lesions. Considering a possible diagnosis of left posterior semicircular canal benign paroxysmal positional vertigo, we performed left Epley's maneuver. She was prescribed diazepam $4 \mathrm{mg}$ for 2 days and $2 \mathrm{mg}$ for the next 3 days, and dimenhydrinate $150 \mathrm{mg}$ for 5 days. Her symptoms improved over the next few days. Seven days later, she visited us again with a complaint of sudden onset of hearing loss on the left side. Fig. 2A shows the pure tone audiometry (PTA) results, which confirmed SSNHL. Currently, she did not report feeling the "whirling" sensation as before, but she did experience disequilibrium-like dizziness. The electrocardiogram, chest radiograph (posteroanterior view), and laboratory results showed no abnormalities. On serology, immunoglobulin $\mathrm{G}$ ( $\mathrm{IgG}$ ) titers were positive for cytomegalovirus (CMV) (titer, 16.7; normal range: $<0.9$ ) and varicella-zoster virus (VZV) (titer, 2.78; normal range: $<0.9$ ). Immunoglobulin $\mathrm{M}$ (IgM) for either virus was not detected, and test results for fluorescent antinuclear antibodies (FANA) were negative. Temporal MRI showed no specific abnormal findings (Fig. 3). She was admitted and administered oral high-dose steroid therapy. She received methylprednisolone $48 \mathrm{mg}$ for 7 days, which was then tapered over 4 days. During the initial 5 days of admission, she did not show any improvement in her hear-

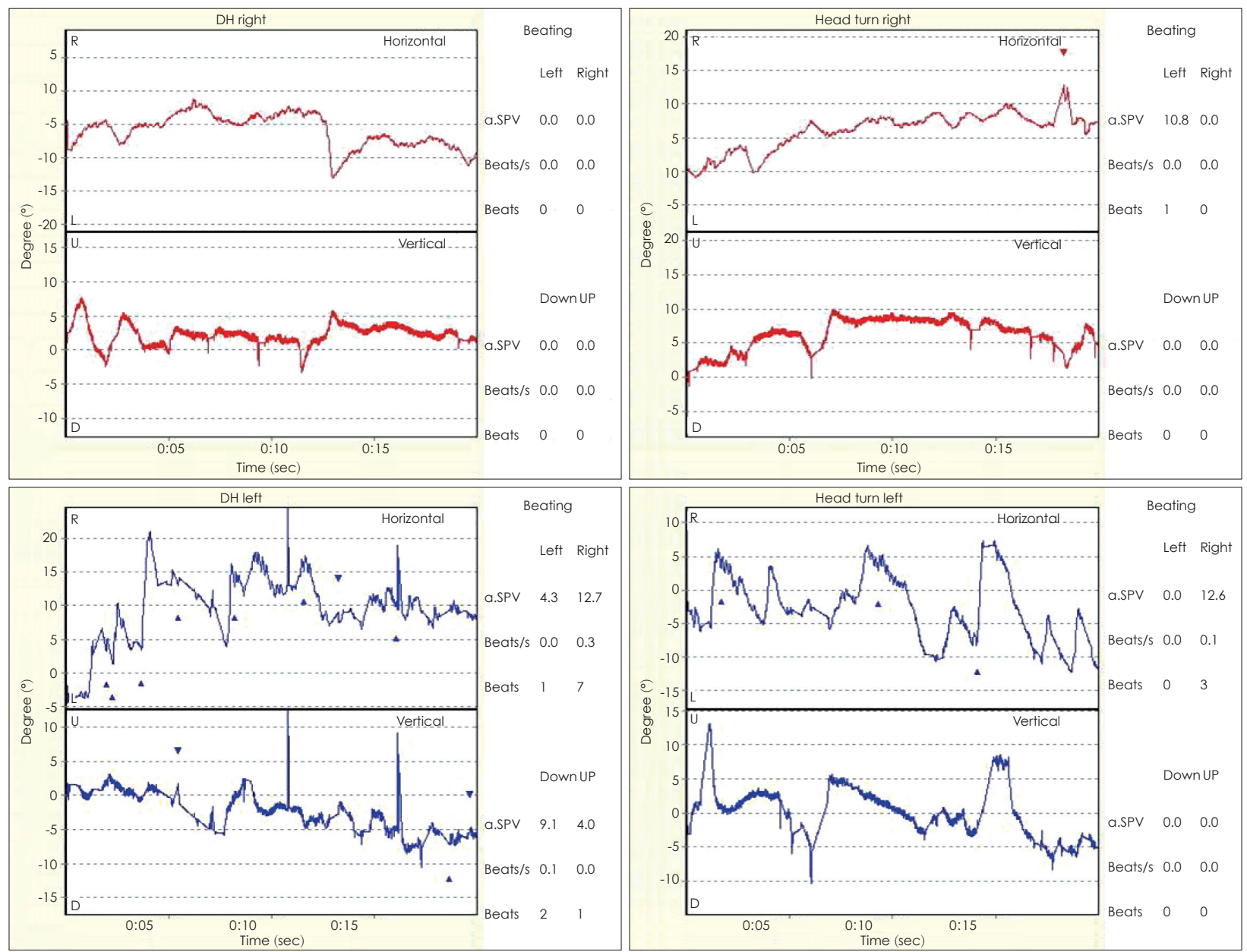

Fig. 1. Videonystagmography performed during the patient's first consultation. DH, Dix-Hallpike position; a.SPV, average slow phage velocity. 


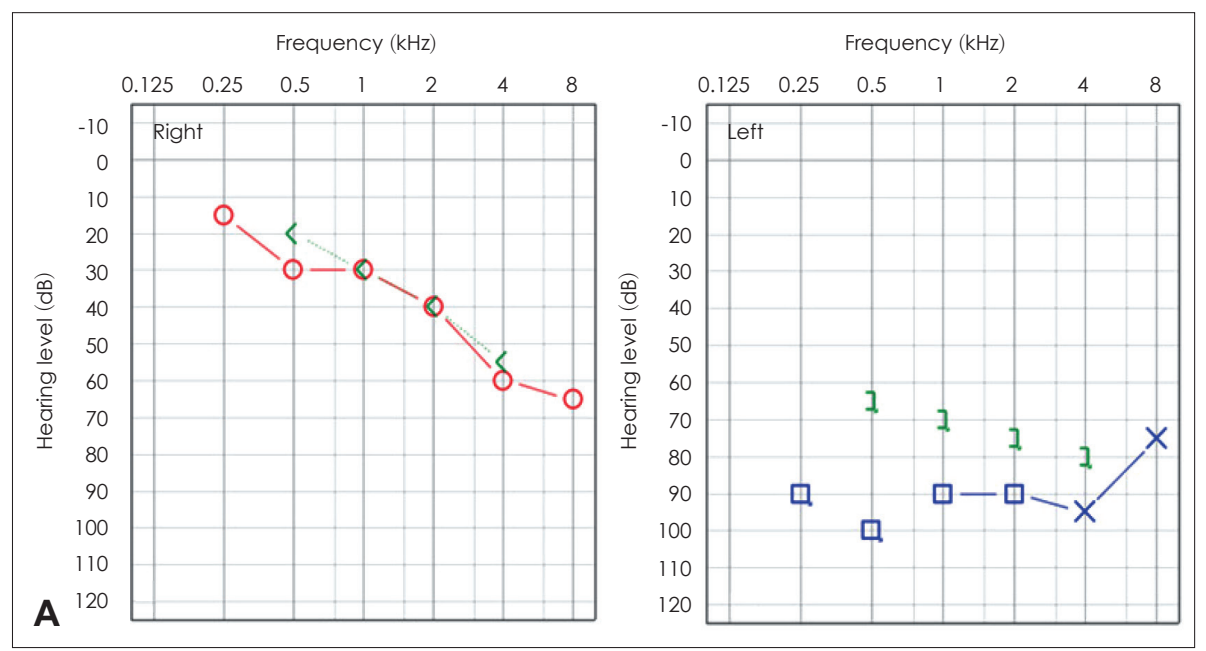

Fig. 2. Pure tone audiometry report after the onset of sudden sensorineural hearing loss (SSNHL) on the left side (A) and sequential SSNHL on the contralateral (right) side (B).

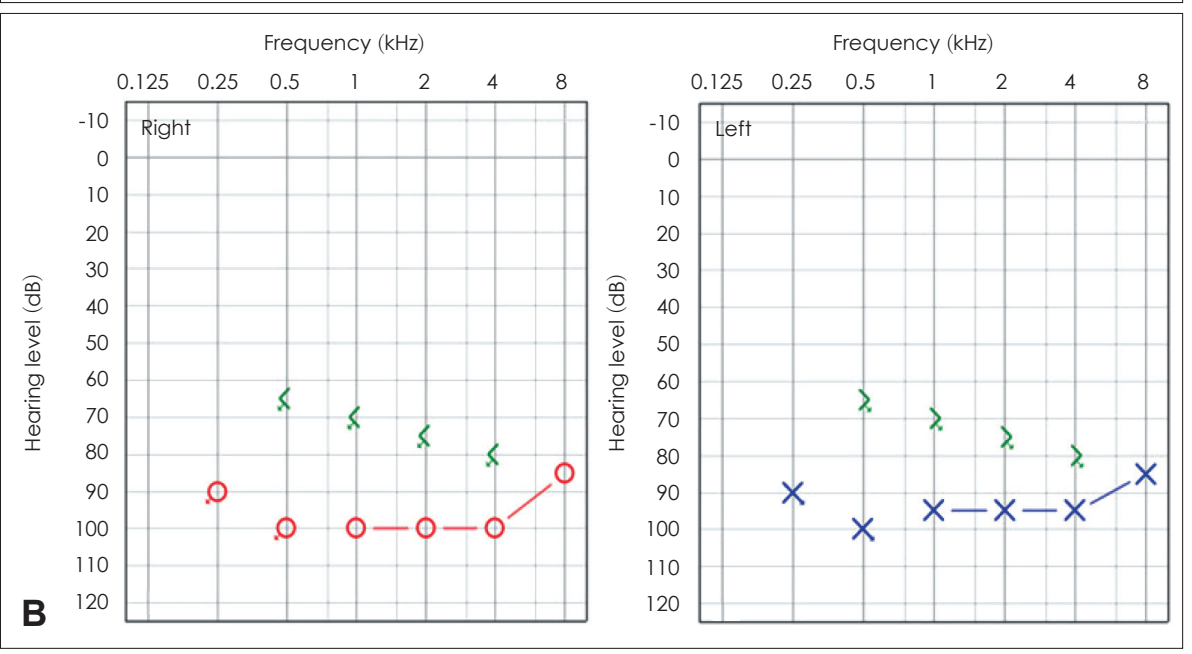

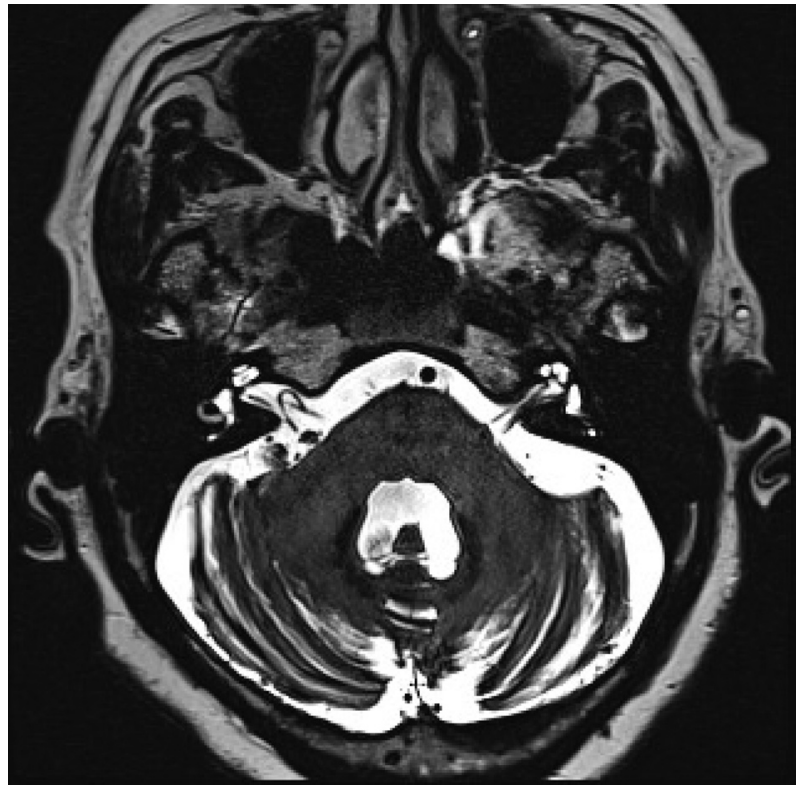

Fig. 3. Temporal magnetic resonance image. ing on the left side. Therefore, intratympanic dexamethasone injection $(5 \mathrm{mg} / 1 \mathrm{~mL})$ was administered into the left ear eight times from day five of hospitalization. However, there was still no improvement in hearing. We explained the general course and prognosis of SSNHL, clarifying that the condition may not resolve, and discharged her. After 37 days of the initial onset of SSNHL (left side), she reported to us again with a complaint of a sudden decrease in hearing on the contralateral (right side). She experienced difficulty in communicating with others. PTA and a test for auditory brainstem responses revealed BSSNHL (Figs. 2 and 4). During all her consultations, her tympanic membrane was intact on otoscopic examination, and she did not complain of any other symptoms except for auditory discomfort and dizziness. We repeated the oral steroid therapy with methylprednisolone (48 mg). Simultaneously, intratympanic dexamethasone injection $(5 \mathrm{mg} / 1 \mathrm{~mL})$ was administered into the right ear five times. However, she showed no improvement on PTA during this admission. The patient wanted to transfer to a tertiary center; therefore, she was discharged from the hospital and referred to a tertiary 

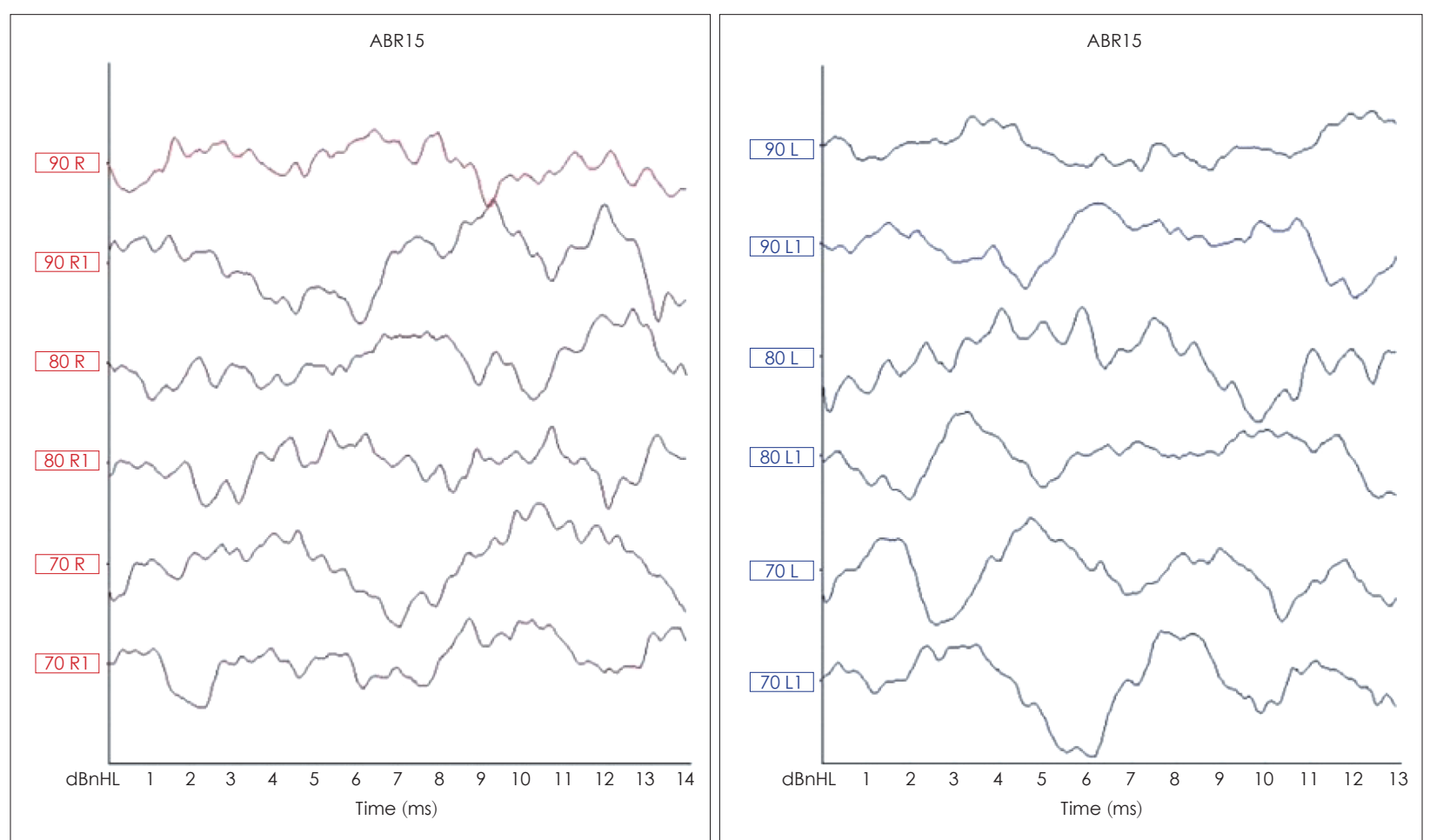

Fig. 4. Auditory brainstem response (ABR) test demonstrating bilateral lack of response.

medical center for further evaluation and treatment. After 4 months, she did not show any improvement at both hearings, and bilateral cochlear implantation surgery was performed. At present, she has not manifested or complained of any newonset of systemic symptoms for general consideration. She can now perform daily activities with a Categorical Auditory Performance score of 5 with a cochlear implant device.

This report was approved by the Institutional Review Board of the Busan Saint Mary's Hospital (BSM2021-04), and the requirement of informed consent was waived.

\section{Discussion}

SSNHL is not a distinct disease but rather a symptom of cochlear injury resulting from several possible causes such as viral inflammation, vascular occlusive disease, or rupture of intra-labyrinthine membranes. Management of SSNHL remains a great challenge for physicians, as the etiology of the condition is unclear, and treatment remains controversial [6]. In general practice, clinicians determine the possible etiology in each patient with SSNHL, based on reported medical history and laboratory findings. If a possible cause cannot be identified, the condition is considered idiopathic.

Patients find SSNHL frustrating and frightening, especially if the hearing loss is bilateral and profound. SSNHL affecting both ears sequentially can be equally frustrating and disheart- ening for the treating doctor as well, and in a busy clinical practice, most otologists tend to focus on the management of the ipsilateral hearing loss reported by a patient during the initial presentation. They may overlook the possibility of sequential occurrence of auditory impairment on the contralateral side since BSSNHL is relatively rare compared to USSNHL. Therefore, they may miss forewarning an affected patient regarding this rare possibility. This could adversely affect the rapport between the patient and the treating doctor, despite the best treatment being offered since the onset of SSNHL.

Before 2007, the classification of BSSNHL as "simultaneous" or "sequential," based on the time interval between onset of hearing loss on each side, was not defined, and the disorder was regarded as a single entity. Xenellis, et al. [2] first defined "simultaneous" and "sequential" SSNHL as that affecting both ears in $<3$ days and 3 days to 1 -month time-interval, respectively. They also reported 7 cases of sequential BSSNHL in 2007 [3,4]. Two of these patients had suffered profound hearing impairment $(>70 \mathrm{~dB})$. However, the report did not describe a relationship between the sudden hearing loss with any definitive past illness or associated underlying pathology in either of these cases; therefore, it is unclear if these cases can be termed idiopathic or non-idiopathic. Some authors included the case of more than 3 months interval as sequential $[3,5,7]$.

It is unknown whether the pathophysiology of sequential BSSNHL differs from that of simultaneous BSSNHL. We could 
hypothesize that certain insults or factors could adversely impact the cochlea bilaterally, making the course of the disease simultaneous in nature. Furthermore, these factors could continue to progress with a more sequential pathological course. If the onset time interval is longer, we could consider the possibility that different kinds of insults affected each side. Wang, et al. [3] defined "successive SSNHL" as that presenting with a time interval of $>1$ year between the onset of hearing loss on each side. They also reported that patients with successive SSNHL have a more severe disease course and a poorer prognosis than that observed in patients with BSSNHL.

We searched the PubMed database for similar reports from 2007 to 2020 (search parameters: Bilateral sudden sensorineural hearing loss) and found no other reported cases of sequential profound BSSNHL. Bing, et al. [7] define sequential BSSNHL as that occurring with an interval of over 3 days, without an upper limit. They reported 46 cases of sequential BSSNHL and 18 cases of sequential, profound hearing loss ( $>80 \mathrm{~dB}$ ). In their report, the median day of onset interval was 808 days, and the first quartile (Q1) was 81.5 days.

Fetterman, et al. [8] described that patients with BSSNHL tend to be older, have pre-existing cardiovascular disease, and are more likely to have a positive antinuclear antibody titer. Oh, et al. [9] reported that BSSNHL demonstrated a strong association with pre-existing conditions such as diabetes mellitus and hypercholesterolemia. While our patient was of an older age with a history of hypercholesterolemia, we do not believe these to be the causative factors for her BSSNHL. The patient's FANA titer was negative, and it was difficult to prove that she had an autoimmune disease. The finding of positive titers for both IgG CMV and IgG VZV was not enough to establish a viral etiology in our patient since most adults acquire these infections during childhood and are therefore seropositive for IgG. Merchant, et al. [10] reported that approximately $90 \%$ and $70 \%$ of the adults in the general population were seropositive for VZV and CMV, respectively.

Our patient experienced dizziness during the treatment period. Her habitual self-administration of diazepam and nutritional supplementation could indicate the possibility of a toxic etiology. Sara, et al. [6] have described that benzodiazepines can be toxic agents and could contribute to the etiology of BSSNHL. However, there is no report on diazepam exerting a definitive toxic effect on cochlear hair cells. Diazepam has even been used to relieve anxiety in patients with SSNHL [11].

There were certain limitations to our assessment of the patient. First, we could not completely evaluate the patient's dizziness using computerized dynamic posturography or a head impulse test. Second, we were unable to gather a detailed medication history to determine any association with her symptoms.
Finally, autoimmune laboratory investigations such as thyroid function test, rheumatoid factor level estimation, and fluorescent treponemal antibody absorption test could not be performed to elucidate a possible definitive cause of the SSNHL.

To conclude, otologists need to be aware of the possibility of sequential BSSNHL, which will allow them to gather a more detailed medical history when the patient first presents with USSNHL, as well as enable them to forewarn a patient about the remote possibility of BSSNHL. Our report describes how BSSNHL could manifest after a prolonged interval without any specifically associated signs, and increased reporting of such cases may help elucidate possible etiological factors in future research.

\section{Acknowledgments \\ None}

\section{Conflicts of interest}

The authors have no financial conflicts of interest.

\section{Author Contributions}

Conceptualization: Joo Young Woo, Byungwoo Yoon. Data curation: Geun Hyung Park, Chang Lok Ji. Funding acquisition: Byungwoo Yoon. Investigation: Joo Young Woo. Supervision: Byungwoo Yoon. Writing — original draft: Joo Young Woo. Writing — review \& editing: Byungwoo Yoon. Approval of final manuscript: all authors.

\section{ORCID iDs}

Joo Young Woo

Chang Lok Ji

Geun Hyung Park

Byungwoo Yoon

https://orcid.org/0000-0002-5972-5714

https://orcid.org/0000-0002-6969-531X

https://orcid.org/0000-0001-9444-8321

https://orcid.org/0000-0001-7870-7493

\section{REFERENCES}

1) Schreiber BE, Agrup C, Haskard DO, Luxon LM. Sudden sensorineural hearing loss. Lancet 2010;375:1203-11.

2) Xenellis J, Nikolopoulos TP, Stavroulaki P, Marangoudakis P, Androulakis M, Tsangaroulakis M, et al. Simultaneous and sequential bilateral sudden sensorineural hearing loss: are they different from unilateral sudden sensorineural hearing loss? ORL J Otorhinolaryngol Relat Spec 2007;69:306-10.

3) Wang Y, Zhang L, Zhang J, Zhang X, Zhang W, Chen X, et al. The clinical analysis of bilateral successive sudden sensorineural hearing loss. Eur Arch Otorhinolaryngol 2016;273:3679-84.

4) Zhang X, Weng Y, Xu Y, Xiong H, Liang M, Zheng Y, et al. Selected blood inflammatory and metabolic parameters predicted successive bilateral sudden sensorineural hearing loss. Dis Markers 2019;2019: 7165257.

5) Oh YM, Oh DH, Jeong SH, Koo JW, Kim JS. Sequential bilateral hearing loss in multiple sclerosis. Ann Otol Rhinol Laryngol 2008; 117:186-91.

6) Sara SA, Teh BM, Friedland P. Bilateral sudden sensorineural hearing loss: review. J Laryngol Otol 2014;128 Suppl 1:S8-15.

7) Bing D, Wang DY, Lan L, Zhao LD, Yin ZF, Yu L, et al. Comparison between bilateral and unilateral sudden sensorineural hearing loss. Chin Med J (Engl) 2018;131:307-15.

8) Fetterman BL, Luxford WM, Saunders JE. Sudden bilateral sensorineural hearing loss. Laryngoscope 1996;106:1347-50. 
9) Oh JH, Park K, Lee SJ, Shin YR, Choung YH. Bilateral versus unilateral sudden sensorineural hearing loss. Otolaryngol Head Neck Surg 2007;136:87-91.

10) Merchant SN, Durand ML, Adams JC. Sudden deafness: is it viral? ORL J Otorhinolaryngol Relat Spec 2008;70:52-60.
11) Gao G, Qian MH, Ji C, Yao M. The analysis of the effect of psychointervention combined with diazepam on patients with sudden sensorineural hearing loss and anxiety. J Clin Otorhinolaryngol Head Neck Surg 2018;32:753-7. 